NASA Technical Memorandum 89926

AIAA-87-9353

\title{
Control Considerations for High Frequency, Resonant, Power Processing Equipment Used in Large Systems
}

(AASA-TH-89926) (CNTECL CCNSILEEATIONS POB

EIGH FREQUENCY, RESONADI, FCSEE FEOCESSILG EQUIEBENT USEL IA LABGE SYSIELS (NASA) 8 p Avail: NIS BC AC2/AF A01 CSCL 10B

N87-23690

$\begin{array}{ll}\text { G3/20 } & \text { Unclas } \\ 0076216\end{array}$

J.W. Mildice and K.E. Schreiner

General Dynamics/Space Systems Division

San Diego, California

and

F. Wolff

Lewis Research Center

Cleveland, Ohio

Prepared for the

22nd Intersociety Energy Conversion Engineering Conference cosponsored by the AIAA, ANS, ASME, SAE, IEEE, ACS, and AIChE Philadelphia, Pennsylvania, August 10-14, 1987 


\title{
Control Considerations for High Frequency, Resonant, Power Processing Equipment Used in Large Systems
}

\author{
Authors: \\ J. W. Mildice; K. E. Schreiner \\ General Dynamics / Space Systems Division \\ San Diego, California \\ F. Wolff \\ NASA / Lewis Research Center \\ Cleveland, Ohio
}

\section{ABSTRACT}

This paper specifically addresses a class of resonant power processing equipment designed to be used in an integrated, high frequency ( $20 \mathrm{KHz}$ domain), utility power system for large, multi-user spacecraft and other aerospace vehicles. It describes a hardware approach, which has been the basis for parametric and physical data used to justify the selection of high frequency $A C$ as the PMAD baseline for the Space Station.

Heated discussions during the process of that selection have shown that there is a general lack of understanding in the Aerospace Industry about the operation of resonant equipment, when used as direct-output inverters and the various converters associated with a large, complex system. This paper is part of a larger effort undertaken by NASA and General Dynamics to be sure that all potential Space Station contractors and other aerospace power system designers fully understand and can comfortably use this technology, which is now widely used in the commercial sector.

In this paper, we will examine control requirements, stability, and operational modes; and their hardware impacts from an integrated system point of view. The current Space Station PMAD system will provide the overall requirements model to develop an understanding of the performance of this type of system with regard to:

- Regulation

- Power Bus stability and voltage control

- Source impedance

- Transient response

- Power Factor effects

- Limits and overloads

Where appropriate, analytical models and conclusions are supported by experimental results from existing equipment.

\section{Historical Perspective}

Early developments in resonant power processing technology (Refs.1 and 2) had limitations which usually eliminated them from consideration as candidates for direct sources of $A C$ power. And for good reasons:

- The frequency was dependent on resonant circuit parameters, (inductances and capacitances) and therefore not very stable or well controlled.

- Regulation was initially accomplished in a pre- or post-processor, with the attendant added losses or waveform degradation.

- Later developments, using clocked frequency control, produced higher distortion waveforms from the delays required to prevent switch overlap, and varied the frequency for output regulation control.

Therefore, most often, the resonant $A C$ produced was used as internal high frequency energy in a more complex configuration. That configuration usually included a post processing stage, which "steered" or "counted down" the individual pulses to produce the desired DC or low frequency AC output. The only real difference between this implementation and the more usual switching converter is that the $A C$ was in the form of sine waves from a resonant $L C$ network, rather than square waves from a semiconductor switch.

This resonant approach found favor in large, high power equipment designs for two reasons:

1. The action of the resonant $A C$ allowed thyristors to commutate themselves off, making that family of high power devices available to high frequency processor designers.

2. Since switching occurred at zero current, the usual dynamic switching losses were virtually eliminated, allowing significant improvements in processor efficiency. 
When system level trades showed that there was the potential for significant life cycle cost and mass savings in high frequency power systems for large, high power, multi-user spacecraft, it was reasonable to investigate whether or not the high frequency could be produced with resonant networks, to gain the additional advantages listed above.

\section{Resonant Processor Requirements}

Two of the most important characteristics necessary for the AC "utility" power system of a spacecraft of the type in question, (such as the NASA Space Station) are frequency control and stability, and the ability to regulate the bus voltage without degrading the waveform. Both are clearly problems for classical resonant power generation.

These, and a few others, provide the major requirements for the design of practical hardware for direct generation of high frequency $A C$ power, using resonant power processing. The important ones are summarized below:

1. Fixed frequency with high stability

2. High efficiency regulation, which does not degrade the output waveform or change output frequency

3. Maintenance of "underdamped" resonant generation networks, over a wide range of load values and power factors.

4. Operates at no load

5. Tolerates overloads and short circuits

6. Modular design, with the ability to parallel sources

\section{Hardware Design and Operation}

\section{Inverter Control}

The NASA and General Dynamics development program has provided simple resonant technology concepts to solve both of the major classical circuit limitations, and also meet the other broad requirements, some of which had not been previously addressed and were thought to be very difficult for resonant configurations.

First, in order to provide a voltage source, with good load regulation and open circuit performance, we chose a "Mapham" (Ref.1) derived driver (inverter) configuration, rather than the more usual "Schwarz" (Ref.2) current source design. This allows for the satisfaction of requirement (4), and the inherently low output impedance provides for excellent load regulation, without the need of the usual feedback loops. However, since the configuration has no inherent line regulation, active $A C$ output voltage control is still required.

In addition, since the equivalent circuit of paralleled modules of this configuration reduces to paralleled inductive sources feeding a single capacitor, they share outputs very well and also satisfy requirement (6) on a first order basis.

Methods for operation of this configuration from a fixed clock, with no waveform degradation due to crossover distortion, have also been well established. (See figure 1.) Antiparallel diodes, CR1 through $C R 4$, allow energy stored in the resonant capacitor $(\mathrm{C} 1)$ to create reverse currents after resonant zero crossing. Therefore, current flows during the time normally reserved for primary power device turn-off and safe operation of the bridge switches without overlap. The natural resonant frequency of the circuit can be significantly higher than the clock frequency without seriously effecting the output waveform, to meet requirement (1) without additional complications.

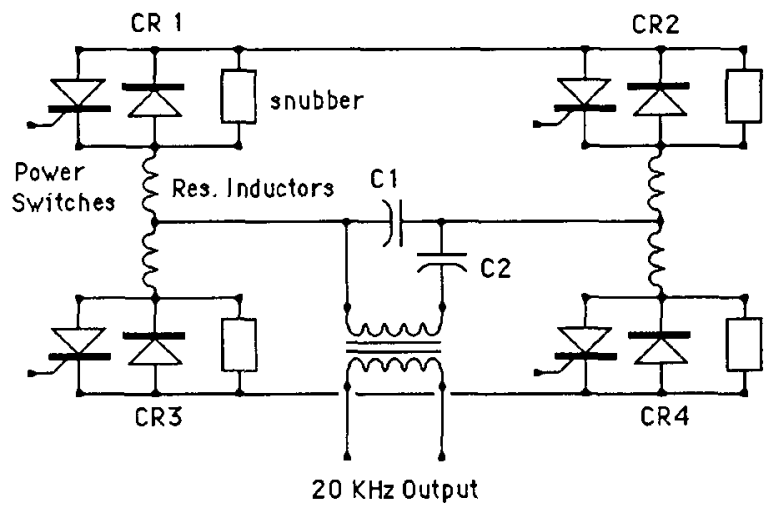

Figure 1 - Basic Resonant Inverter

However, requirements (3) and (5) are the obvious stumbling blocks for this parallel output topology. When the usual configuration is loaded beyond the resonant circuit critical damping point, the switches do not commutate off in time to maintain good frequency control without overlap. The alternative of forced commutation adds distortion and switching losses, and eliminates conventional thyristors as power switch options. 
The addition of a series output capacitor (C2) provides a good, simple solution. Now, since any load, or overload (including a short circuit), is in series with the output capacitor, it can never cause the primary resonant circuit to be overdamped, and it will always operate properly. It is also important to note that $\mathrm{C} 2$ does not have a detrimental effect on output impedance.

When we shifted the primary circuit resonant frequency higher than the clock frequency, that frequency moved to the inductive side of the impedance curve. If $\mathrm{C} 2$ is properly sized, it will cancel this inductance and lower the output impedance.

This leaves the last remaining requirement (2), active regulation. Our approach, which maintains the fidelity of the output waveform and does not add additional losses, is called Phasor Regulation. (See figure 2.) Simply stated, it is the addition of single frequency, sinusoidal $A C$ voltages, with controlled phase differences. The resulting output is an undistorted sinusoid of the same frequency, having the following relationships to the original power outputs:

since:

$$
[A] \sin w t+[B] \sin (w t+\varnothing)=
$$$$
[(A+B) \cos (\varnothing / 2)] \sin (w t+\varnothing / 2)
$$

therefore:

Amplitude of summed output $=(A+B) \cos (\varnothing / 2)$

Phase of summed output $=\varnothing / 2$

Frequency of summed output $=w t$

where: $\quad A=$ amplitude of signal 1

$\mathrm{B}=$ amplitude of signal 2

$w t=$ frequency of operation

$\varnothing=$ phase diff. between the outputs

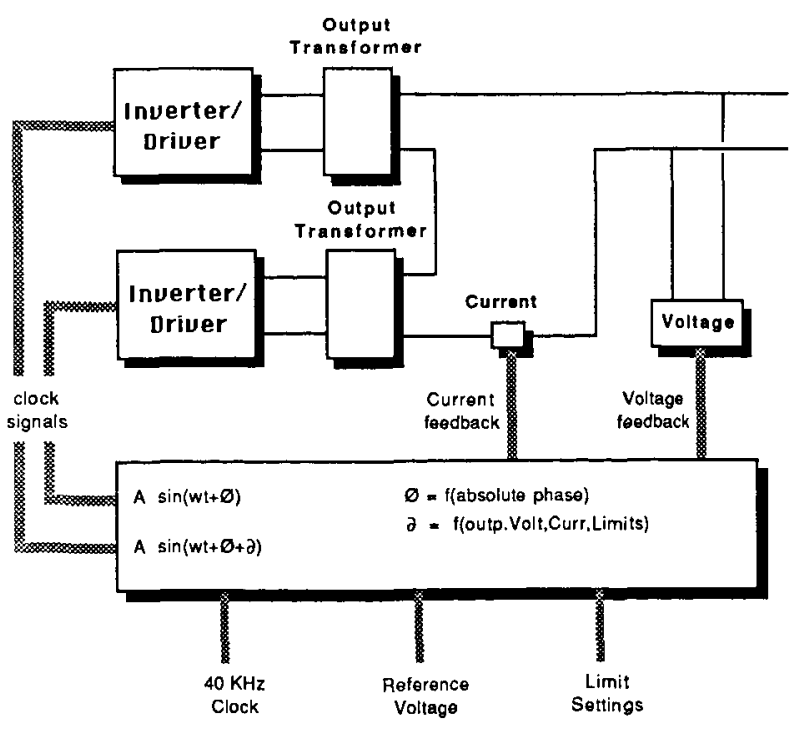

Figure 2 - Inverter Phasor Regulation
Therefore, the desired output amplitude control can be accomplished by adding the outputs of two resonant inverter modules in series (as shown in figure 2) and controlling the phase difference between them, by adjusting the relative phases of their respective external clock signals.

The actual control hardware is a variable analog phase shifting network, placed in series with the power module clock signals. The error signal used to control the phase shifter is derived from the sum of the output voltage feedback and a computer-generated reference signal. A similar current feedback loop is used to provide load dependent output impedance control to enhance output current sharing of paralleled modules with active control. Finally, a higher gain current feedback loop overrides the voltage loop when when its reference is exceeded, providing non-dissipative current limiting from the basic phasor regulator.

\section{Power Transmission Control}

Because of the high frequency $(20 \mathrm{KHz})$ selected for power distribution on the NASA Space Station, and previous difficulties experienced in designing $400 \mathrm{~Hz}$ power distribution systems on some recent large, commercial aircraft programs, we have focussed early attention on transmission system control.

Since power losses cost so much on large space vehicles, the power transmission system must also be designed for low losses, to meet the same level of requirements as the rest of the power processing equipment. Inductance and capacitance of long lines cannot be totally eliminated, and this leads to underdamped LC distributed networks, which must be controlled with the same care as other system elements.

While $20 \mathrm{KHz}$ is a higher frequency than we have previously considered in power transmission, the longest distances with which we must deal are on the order of 100 to 150 meters. These distances are very short compared to the $20 \mathrm{KHz}$ wave length (approxmately 15 kilometers) and high frequency transmission line effects such as reflections and standing waves are of no concern. Therefore, we can confidently model these lines as multiple sections of lumped elements, and define the element parameters from test data on currently constructed engineering articles.

Analytical predictions and test data from a flat "stripline" type engineering evaluation model power bus have yielded the parameters shown below (for a $50 \mathrm{~m}$. line). 


$$
\begin{aligned}
& R=0.065\left(10^{-3}\right) \text { Ohms } \\
& L=1.9\left(10^{-6}\right) \text { Henries } \\
& C=69\left(10^{-3}\right) \text { Microfarads }
\end{aligned}
$$

Computer analysis of lumped parameter line models composed of ten meter sections, with resistive and reactive loads connected at various locations, shows that unloaded and lightly loaded line voltages deviate from nominal, but are stable and well behaved, even if the input port is the only location for active feedback. Figure 3 shows the envelope of possible line voltages calculated by this analysis. Since this analysis is in direct contradiction with earlier, less rigorous estimates and opinions, data taken on actual fifty meter line samples of a $25 \mathrm{KW}$ bus design was used to confirm it. As shown on figure 3 , the actual performance was even better than our more conservative analysis.

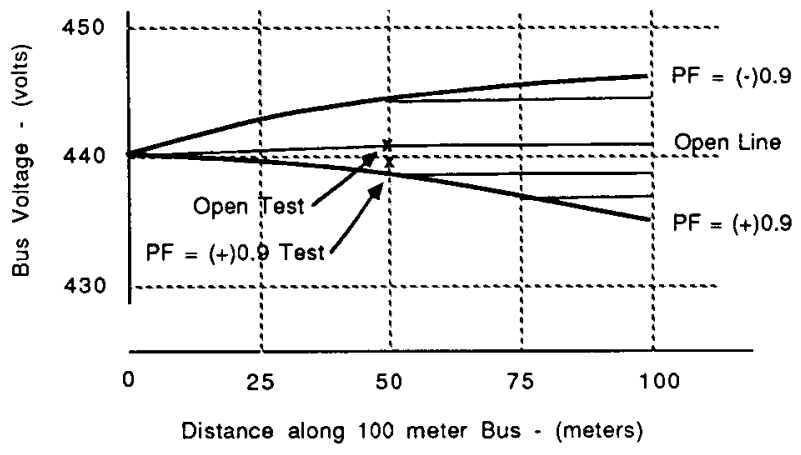

Figure 3 - "Stripline Voltage" Characteristics

Therefore, based on rigorous analysis and actual test data, we can confidently conclude that power distribution bus system performance will be predictable, well behaved and stable for vehicles of typical space station size and complexity; without the need for remote sense inputs to high speed, active control loops or complex load compensation or matching. In addition, we can predict that bus voltage can be maintained to within $\pm 3 \%$ of its nominal value along its entire length without remote feedback.

\section{Inverter Output Impedance Effects}

Source impedance is important in two frequency regimes. First (and easiest to consider) are the low frequency and steady state characteristics, determined by the active control loop and the phasor regulation described above. The loop used contains the familiar integrator type comparator to sum the feedback and reference signals, so the steady state gain approaches the open loop gain of an integrated circuit operational amplifier; and the loop feedback capacitor is sized for a zero $\mathrm{db}$ crossover at approximately $3.0 \mathrm{KHz}$.

At the higher frequencies, the impedance is dominated by the lumped elements in the resonant output network. Figure 4 shows the calculated and measured output impedance in the neighborhood of $20 \mathrm{KHz}$ for the current designs. (The magnitude scale is referred to

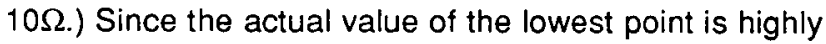
dependent on actual circuit " $Q$ ", which is strongly influenced by hard to define stray resistances, it is difficult to predict its value, except to say that it is below $0.01 \Omega$. Actual data, taken on some ten different inverters of different sizes shows the magnitude of the open loop impedance (at $20 \mathrm{KHz}$ ) in the present designs (with output impedance only partially compensated) is:

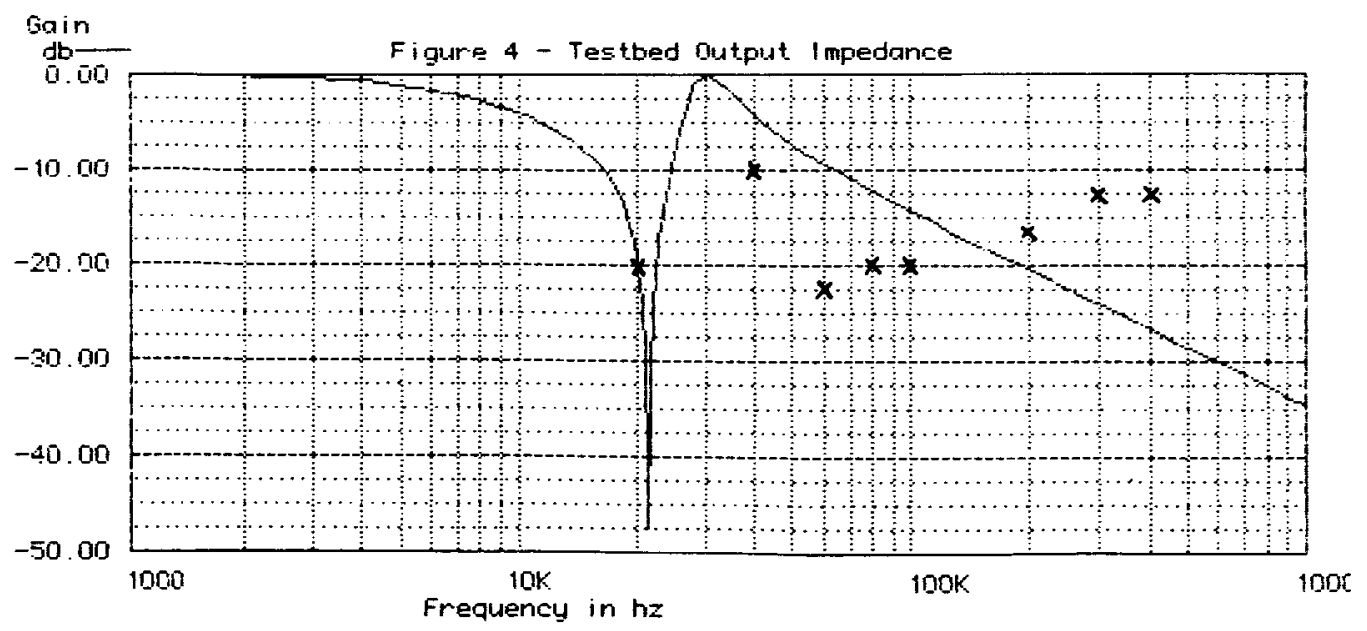




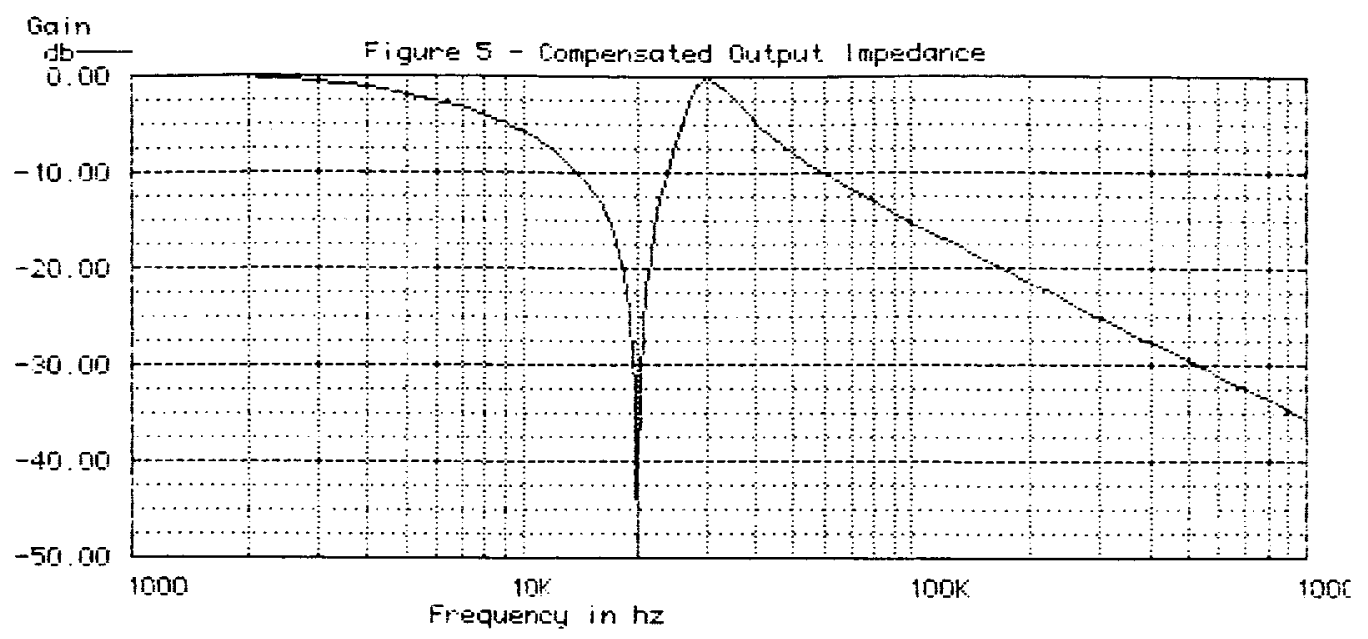

\section{$Z_{0}=1.0 \mathrm{ohm} \pm 50 \%$ (which is consistent with calculated data)}

Figure 5 represents a fully compensated design, where the actual $20 \mathrm{Khz}$ output impedance is minimized. The measured high frequency values also shown on figure 5 are based on voltage effects, caused by induced harmonic currents, on our $25 \mathrm{KW}$ testbed system, operating at full load. They are shown both at the inverter outputs and at the end of a 50 meter power bus. These values demonstrate that the inverters and distribution system have low enough output impedances, over the range of important harmonic frequencies, to meet the mission model space station EMI and power quality needs.

\section{Power Factor Effects}

Because this design has underdamped reactive networks at the inverter outputs, and those networks are so intimately associated with proper inverter operation, first exposure to this approach prompts concern about the effects of reactive (low Power Factor) loads.

Capacitive Loads: As explained above, the series output capacitor isolates capacitive loads, because any value of load capacitor makes the added capacitance seen by the resonant circuit less than the series capacitor, and adding the value of the series capacitor does not disable the resonant operation or cause a commutation failure. However, large capacitive loads exceed the KVA rating of an inverter, just as any other load does, and the active output current limiting protection function of the inverter will reduce the output voltage appropriately, the limit the output power to its preprogrammed value. Therefore, capacitive loads have no effect until they become overloads, where they are controlled the same way as any other overload.

Inductive Loads: The effect of inductance on the output is more complex. Since inductive reactance can be thought of as negative capacitive reactance, there is a point where an inductive load effectively cancels the series output capacitor. (The point where the two components are resonant at $20 \mathrm{KHz}$.) Even larger values of inductance then "subtract" from the resonant capacitor, increasing the natural frequency of the network, causing added distortion. If the capacitance gets small enough, it could ultimately cause a commutation failure, if there were no active current limiting. Typical inverter designs will place this series resonant point at a power factor of about 0.7 when fully loaded (lower with lighter loads). Even though it takes a much lower power factor (below 0.5 at full load) to cause a commutation failure, we usually fix the spec limit at 0.7 , since that point results in no shift in the resonant network natural frequency, and therefore, no increase in output distortion.

\section{Limits and Overloads}

Since several of the previous subjects refer to active limiting and overload control, it is important to understand the controls for this aspect of inverter operation. As explained in Section 4, There are two active current feedback loops. The first is used to control output impedance for proper current sharing from different, regulating, paralleled sources. The second is a high gain loop which has no output until its preset current threshold exceeded. When it is exceeded, the phasor regulator shifts to a constant current mode, and reduces the inverter output to prevent a catastrophic failure (in the inverter or bus system). 
That preset value is a data bus transmitted digital word reference, and therefore fully adjustable by the system computer. Therefore, three operational modes are possible when an overload or fault is present, each under conscious control with no inadvertent "smoke and fire".

1. We (or the system computer) can elect to leave the control in place and shift critical loads to another bus.

2. We can elect to raise the limit and try to continue to power the loads on the overloaded bus.

3. In some extreme emergency, we can elect to raise the limit to the capability of the basic source or to a level likely to burn out the fault, and attempt to clear it.

\section{Conclusions}

$20 \mathrm{KHz}$ power system technology is a mature technology, both from the point of view of analytical understanding, and the design, construction, and test of second generation hardware, as evidenced by the $25 \mathrm{KW}$ testbed system now delivered to NASA LeRC for their Space Station Power System test bed. Many simple circuit developments and extrapolations have carried resonant generation topologies into practical utility power system sources. Analysis and test have shown that this system approach is both practical and easy to implement for a Space Station type vehicle, and it contributes significantly to improved operation and efficiency.

\section{References}

1. "An SCR Inverter with Good Regulation SineWave Output"; Neville Mapham, IEEE Transactions on Industry and General Applications; IGA-3, No. 2, Apr-May, 1967

2. "Bidirectional Four Quadrant (BD4Q) Power Converter Development"; Final Report, NASA CR159660, F.C.Schwarz, Power Electronics Assoc. Inc.

3. "Bidirectional Power Converter Control Electronics"; Final Report, NASA CR-175070, J.W.mildice, General Dynamics/Space Systems Division 


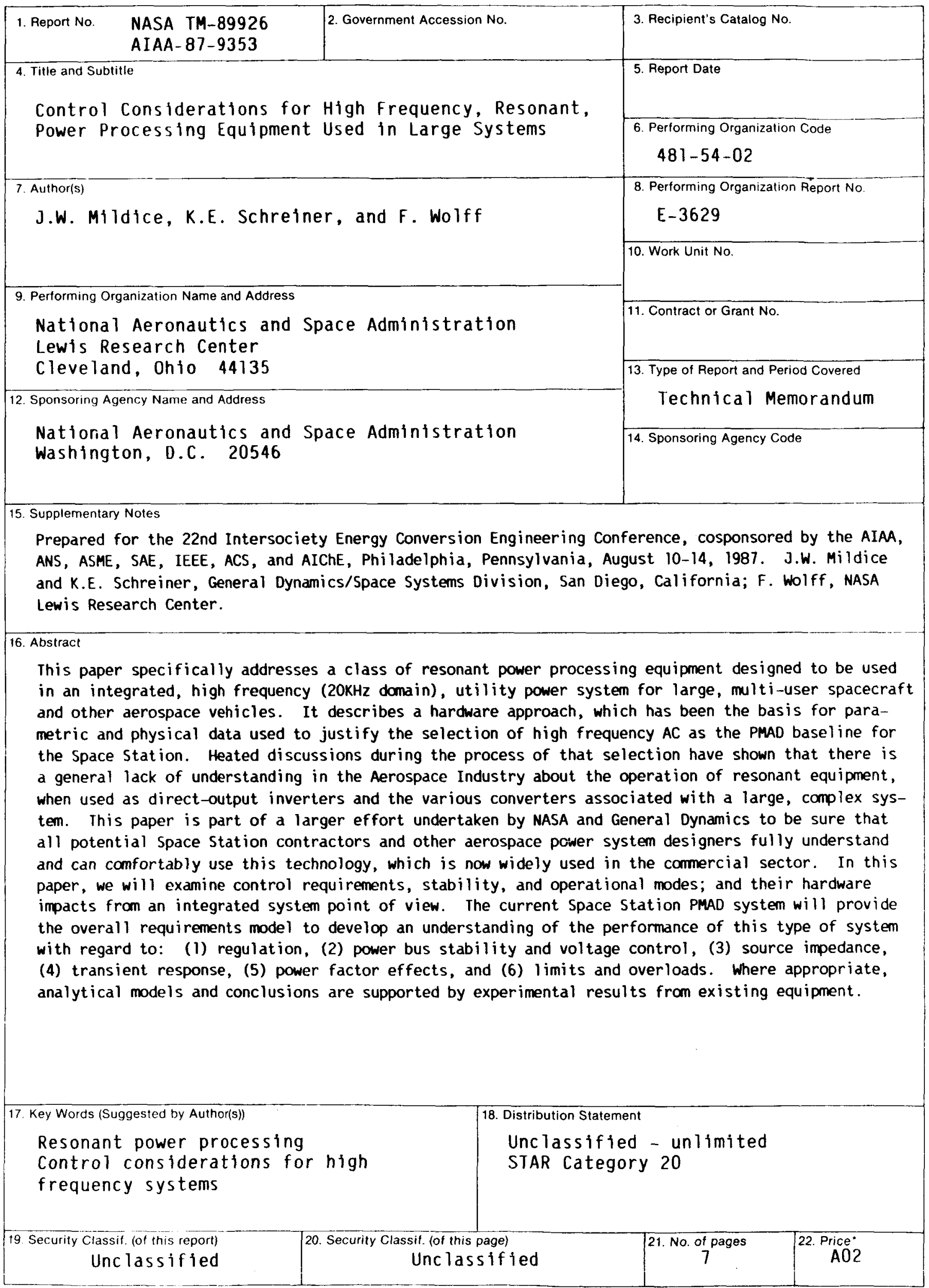

*For sale by the National Technical Information Service, Springfield, Virginia 22161 